\title{
Influence of polyphenols on in vitro starch digestibility of oat porridges
}

\author{
P. S. Thondre, L. Ryan and C. J. K. Henry \\ Functional Food Centre, School of Life Sciences, Oxford Brookes University, Gipsy Lane, Headington, \\ Oxford OX3 OBP, UK
}

Polyphenols were found responsible for reduced glycaemic response to carbohydrate foods, especially legumes ${ }^{(1)}$. The objective of the present study was to investigate the effect of polyphenols in oat porridges on their in vitro digestibility. Oats have gained considerable attention in recent years as a good source of soluble fibre, antioxidants such as vitamin E, various phenolic compounds and avenanthramides ${ }^{(2)}$. The main source of oats in the UK diet is breakfast cereals representing more than $60 \%$ of oat products sold. Sixteen commercially available oat porridges were subjected to in vitro digestion ${ }^{(3)}$. The amount of rapidly digested starch (RDS) was measured in the samples drawn before digestion, at the end of gastric phase and after 20,60, 120 and 180 min during intestinal phase ${ }^{(4)}$. Polyphenol contents of oat porridges were measured by the Folin-Ciocalteau method ${ }^{(5)}$ and expressed as gallic acid equivalents (GAE).

All the oat porridges had significant amounts of polyphenols ranging from 1500 to $1850 \mu \mathrm{g} / \mathrm{g}$ GAE. The oatmeal of Alford (Montgarrie Mill, Alford, Scotland) showed significantly higher polyphenol content than the others $(P<0.05)$. The oat porridges varied in their digestibility. There was a significant $(P<0.01)$ negative correlation between the polyphenol content and RDS $(r=-0.743)$ as shown in this figure. There was no significant correlation between the amount of fibre present in the oat porridges and starch digestibility.

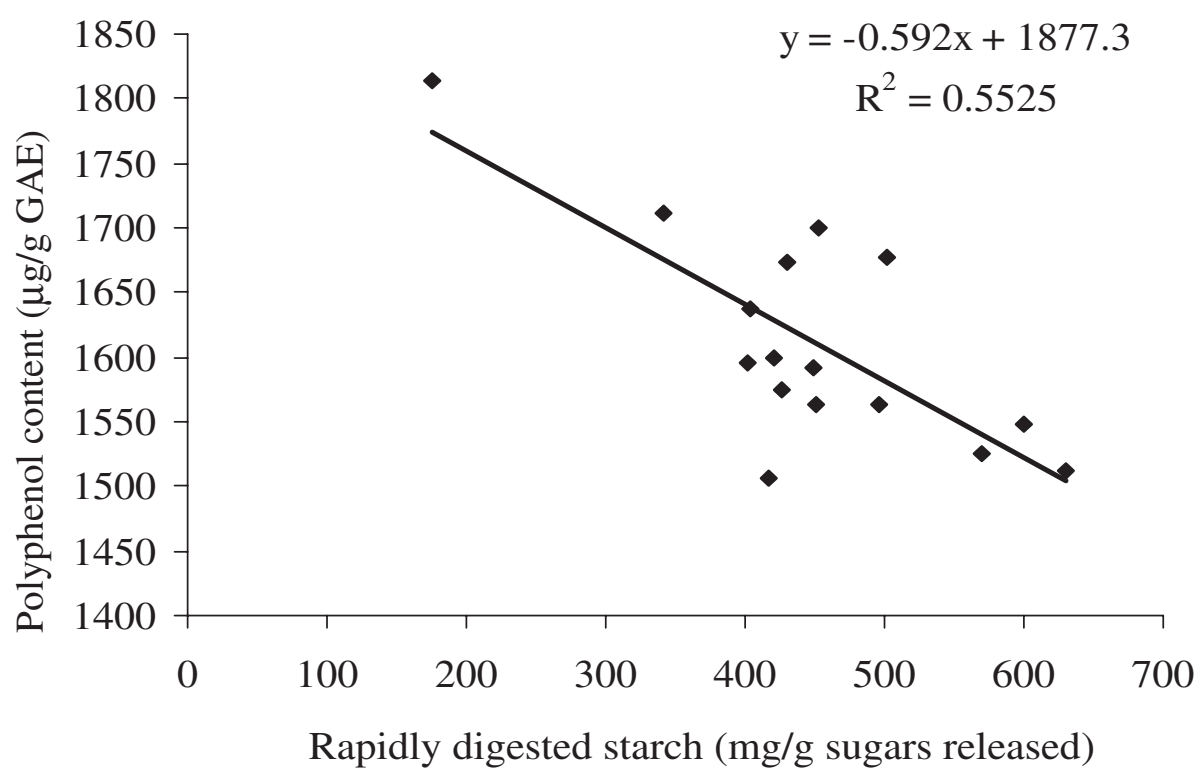

The results presented demonstrate a possible interaction between starch and polyphenols in the oat porridges thereby slowing down the starch digestibility. Another possible mechanism is the interaction between amylase and polyphenols that might have reduced starch digestibility. So far, the beneficial effects of oats porridges on glycaemic response have been attributed to their fibre content, especially $\beta$-glucan ${ }^{(6)}$. This is the first study that reports the effect of polyphenols on starch digestibility of oat porridges. In conclusion, polyphenol content could have potential effect on glycaemic response to oat porridges in vivo.

1. Thompson LU, Yoon JH, Jenkins DJA et al. (1984) Am J Clin Nutr 39, 745-751.

2. Peterson DM, Hahn MJ, \& Emmons CL (2002) Food Chem 79, 473-478.

3. Thondre PS, Monro JA, Mishra S et al. (2010) Food Res Intl 43, 1476-1481.

4. Mishra S \& Monro JA (2009) J Cereal Sci 50, 61-66.

5. Sharma P \& Gujral HS (2010) Food Chem 120, 673-678.

6. Butt MS, Tahir-Nadeem M, Khan MKI et al. (2008) Eur J Nutr 47, 68-79. 